\title{
Mensurando a consciência ambiental do consumidor: um estudo comparativo entre as escalas NEP e ECCB
}

Measuring the consumer environmental concern: a comparative study between the NEP and ECCB scales

Midiendo la conciencia ambiental del consumidor: un estudio comparativo entre las escalas NEP y ECCB

\section{Otávio Freire}

- Doutor em Ciências da Comunicação pela Escola de Comunicações e Artes da Universidade de São Paulo (ECA-USP)

- Professor do Programa de Pós-Graduação em Administração da Universidade Nove de Julho (Uninove)

- Líder do grupo de pesquisa "Comportamento do consumidor no esporte", da Uninove

- Professor do Curso de Marketing da Escola de Artes, Ciências e Humanidades da Universidade de São Paulo (EACH-USP)

- Coordenador assistente do Centro de Estudos em Avaliação e Mensuração em Comunicação e Marketing (Ceacom) da ECA-USP

- E-mails: otfreire@usp.br e otaviofreire@uninove.br

\section{Filipe Quevedo-Silva}

- Doutorando em Administração - Marketing no Programa de Pós-Graduação em Administração da Universidade Nove de Julho (Uninove)

- Mestre em Administração pela Universidade Federal do Mato Grosso do Sul (UFMS)

- Bacharel em Administração pela mesma instituição

- E-mail: admquevedo@hotmail.com

\section{Elias Frederico}

- Doutor e mestre em Administração pela Faculdade Getúlio Vargas - FGV/SP

- Bacharel em Engenharia pela Fundação Educacional Inaciana (FEI)

- Professor-doutor do Curso de Marketing da Escola de Artes, Ciências e Humanidades da Universidade de São Paulo (EACH-USP)

- Professor da Fundação Getulio Vargas (Eaesp-FGV)

- E-mail: efrederico@usp.br 


\section{Resumo}

O presente estudo objetiva comparar e avaliar a adequação de duas escalas, para mensuração da consciência ambiental: New ecological paradigm (NEP) e Ecologically conscious consumer behavior (ECCB). O exame da literatura indicou que uma decorrência da consciência ambiental é a intenção de comportamento de consumo ambiental (ICCA). Para a operacionalização do estudo, foi aplicado um survey on-line com 538 consumidores brasileiros. Foram utilizadas técnicas estatísticas multivariadas para verificar a dimensionalidade das escalas, bem como para avaliar sua associação com a ICCA. Os resultados indicam que a escala ECCB é mais adequada para medir a ICCA.

PALAVRAS-CHAVE: CONSCIENNCIA AMBIENTAL • COMPORTAMENTO DE CONSUMO AMBIENTAL・ESCALAS CONCORRENTES NEP E ECCB

\section{Abstract}

The purpose of this study is to compare and assess the feasibility of two scales, NEP and ECCB, to measure environmental concern. Examination of literature indicated that environmental consumer behavior intention (local acronym ICCA) derives from environmental concern. To render the study operational, an on-line survey was conducted among 538 Brazilian consumers. Multivaried statistics techniques were used to verify the NEP and ECCB scale dimensions, as well as to assess their association with the ICCA. The results indicate that ECCB is more adequate to measure the ICCA.

KEYWORDS: ENVIRONMENTAL CONCERN • ENVIRONMENTAL CONSUMER BEHAVIOR • COMPETING NEP AND ECCB SCALES

\section{Resumen}

Este estudio tiene como objetivo comparar y evaluar la adecuación de dos escalas para la medición de la conciencia ambiental: New ecological paradigm (NEP) y Ecologically conscious consumer behavior (ECCB). La revisión de la literatura indicó que un resultado de la conciencia ambiental es la intención del comportamiento de consumo ambiental (ICCA). Para la operacionalización del estudio, fue aplicado un survey online con 538 consumidores brasileros. Fueron utilizadas técnicas estadísticas multivariadas para verificar la dimensionalidad de las escalas, así como evaluar su asociación con ICCA. Los resultados indican que la escala ECCB es la más adecuada para la medición de ICCA.

PALABRAS CLAVES: CONCIENCIA AMBIENTAL - COMPORTAMIENTO DE CONSUMO AMBIENTAL • ESCALAS COMPETIDORAS NEP Y ECCB 
$\Delta$ s questões relacionadas à sustentabilidade ambiental têm se tornado cada vez mais imporItantes para as empresas (Matos; Hall, 2007). Muitas organizações têm adotado estratégias e lançado produtos com apelo ambiental por perceber que, ao longo do tempo, parte da população não somente se importa com essa temática, mas também tem levado isso em consideração em suas atividades de consumo (Santos et al., 2008).

A relevância do tema também fica evidente ao se analisar a produção científica. Diversos artigos nacionais e internacionais têm estudado, por exemplo, as práticas ambientais adotadas pelas empresas (Mann et al., 2010; Souza, 2002), o consumo de determinados bens que possuam atributos de sustentabilidade (Natali, 2012; Bedante, 2004) as características que diferenciam esses consumidores (Hirsh, 2010; Mostafa, 2007) e a relação do que habitualmente é chamada de consciência ambiental com o comportamento de consumo (Fujii, 2006; Mainieri et al., 1997).

Contudo, apesar da quantidade de trabalhos, nota-se uma grande variabilidade nas escalas utilizadas pelos pesquisadores para se avaliar a consciência ambiental dos consumidores. Alguns desenvolvem escalas próprias, enquanto outros fazem uso de escalas mais genéricas inserindo questões específicas ao objeto estudado, como, por exemplo, questões relacionadas a produtos reciclados (Bedante, 2004). Além disso, ao pesquisar trabalhos que utilizaram uma mesma escala, não é incomum encontrar divergência na quantidade de itens utilizados, seja já no modelo de estudo inicial ou após o tratamento estatístico dos dados.

Em meio a essa diversidade de medidas, uma escala utilizada por muitos pesquisadores é a do New ecological paradigm (NEP), proposta por Riley Dunlap et al. (2000). Além da NEP, outra escala que pode ser encontrada com certa recorrência, principalmente em trabalhos nacionais, é a Ecologically conscious consumer behavior (ECCB), proposta por Robert Straughan e James Roberts (1999). Esta escala, diferentemente da NEP, é voltada às práticas de consumo sustentável, abordando, por exemplo, o consumo de produtos que tenham excesso de embalagem.

Sendo assim, com base na relevância crescente do tema na área de comportamento de consumo e sabendo da importância de se obterem métodos de mensuração eficientes, este artigo tem como objetivo comparar as escalas NEP e ECCB, analisando qual representa melhor a consciência ambiental em relação à intenção de comportamento de consumo ambientalmente responsável da população brasileira.

Nosso estudo está estruturado da seguinte forma: 1. os marcos teóricos que fundamentam a proposição do modelo de pesquisa são estabelecidos; 2. a metodologia de pesquisa é delineada; 3. os resultados são discutidos; e 4. as conclusões, limitações da pesquisa e sugestões para estudos futuros são apresentadas. 


\section{CONSCIÊNCIA AMBIENTAL E AS ESCALAS NEP E ECCB}

A consciência ambiental, segundo Bodo Schlegelmilch, Greg Bohlen e Adamantios Diamantopoulos (1996) é definida como um construto multidimensional composto por elementos atitudinais, comportamentais e cognitivos. Segundo a abrangente revisão de estudos feita por esses autores, para avaliar o nível real de consciência ambiental de um indivíduo, é preciso analisar a sua preocupação ou interesse pelo tema, seus comportamentos passados, atuais e futuros (intenção comportamental) e o quanto ele compreende acerca do impacto de suas ações no meio ambiente.

Com base nas teorias de comportamento planejado e de atitude, diversos estudos (Hartmann e Apaolaza-lbáñez, 2012; Akehurst, Afonso e Gonçalves, 2012; Ishaswini e Datta, 2011) procuram demonstrar a relação entre consciência ambiental e o comportamento do consumidor, sendo que, de maneira geral, sugere-se que, quanto maior é o nível de consciência ambiental do individuo, maior a probabilidade de consumir produtos ambientalmente sustentáveis. Dessa forma, para melhor compreender essas relações - e o próprio consumidor -, é imprescindível mensurar de forma eficiente esse construto. Estudos nessa área têm utilizado diferentes métricas para isso. Contudo, vários pesquisadores operacionalizaram as escalas NEP e ECCB como formas de medir a consciência ambiental. O Quadro 1 apresenta uma síntese das pesquisas publicadas em periódicos nacionais e internacionais.

\section{Quadro 1 - Estudos sobre consciência ambiental}

\begin{tabular}{|l|c|}
\hline \multicolumn{1}{|c|}{ Artigos } & $\begin{array}{c}\text { Escala para } \\
\text { consciência } \\
\text { ambiental }\end{array}$ \\
\hline $\begin{array}{l}\text { Nisbet, Zelenski e Murphy (2009); Poortinga, Steg e Vlek (2004 } \\
\text { e 2002); Casey e Scott (2006); Chung e Poon (2000); Kaiser, } \\
\text { Hubner e Bogner (2005); Thapa (1999); Mostafa (2007); Hart- } \\
\text { mann e Apaolaza-Ibáñez (2012); Arnocky, Dupuis e Stroink } \\
\text { (2012); Mainieri et al. (1997) }\end{array}$ \\
\hline $\begin{array}{l}\text { Ishaswini e Datta (2011); Akehurst, Afonso e Gonçalves (2012); } \\
\text { Braga Junior et al. (2012); Bedante (2004); Natali (2012); Riley, } \\
\text { Kohlbacher e Hofmeister (2012); Cervellon (2012); Zuraidah et } \\
\text { al. (2012); Singh e Gupta (2013); Jain e Kaur (2006); Said et al. } \\
\text { (2003) }\end{array}$ \\
\hline
\end{tabular}

Fonte: Elaborado pelos autores 


\section{A escala NEP}

A crescente preocupação com o meio ambiente moveu alguns pesquisadores em direção à criação de um novo paradigma socioambiental, destacando o papel do meio ambiente na nova interpretação econômica, chamado de Novo paradigma ambiental (Silva Filho et al., 2010). A criação de uma escala para medir esse novo paradigma foi proposta por Riley Dunlap e Kent D. Van Liere em 1978, a New environmental paradigm scale, e revisada em 2000, quando passou a se chamar New ecological paradigm scale (Dunlap et al., 2000).

Segundo os autores, em relação à escala original, as alterações na NEP acarretaram uma ampliação da visão de mundo sob a perspectiva ecológica em função da avaliação conceitual dos termos ambiental e ecológica. Isto acarretou mudanças na sua nomenclatura e foram incluídos itens invertidos na escala, denominados itens anti-NEP. Ocorreram, ainda, atualizações na terminologia empregada nos itens. A escala NEP revista é composta por quinze itens, sendo seis invertidos, ou seja, concordar com estes significaria negar o paradigma. As afirmativas são mensuradas por meio de escala de concordância de cinco pontos (tipo Likert), variando do discordo totalmente ao concordo totalmente.

A criação dessa escala proporcionou o desenvolvimento de diferentes linhas de pesquisa, a exemplo da análise entre ambientalismo, atitudes e comportamentos reais, tais como o consumo de produtos ecologicamente corretos e a reciclagem (Silva Filho et al., 2010). Além do estudo original de Dunlap et al. (2000), onde a validação foi proposta com questões sobre atitudes e opiniões, encontram-se estudos sobre a relação entre NEP e reciclagem (Vining e Ebreo, 1992; Schultz e Oskamp, 1996; Ebreo, Hershey e Vining, 1999), NEP e consumo ecologicamente correto (Roberts; Bacon, 1997); e atitudes pessoais e medidas de ambientalismo (Blake, Guppy e Urmetzer, 1997; Scott e Willits, 1994; Gooch, 1995).

\section{A escala ECCB}

A ECCB foi elaborada inicialmente por James Roberts (1996) a partir de estudos que abordavam variáveis que influenciavam o comportamento socialmente responsável durante o processo de decisão de compra. Para validar a escala, ele realizou uma pesquisa com 582 americanos, considerando que o consumidor ecologicamente consciente era aquele que comprava produtos os quais considerava terem impacto favorável ao meio ambiente. O resultado foi uma estrutura com dois fatores, sendo o primeiro formado por 22 itens e o segundo, por oito. $\mathrm{O}$ autor excluiu o segundo fator e ficou com uma estrutura unidimensional composta por 22 itens.

Em 1999, Roberts se junta a Robert Straughan para replicar seu estudo, buscando verificar a existência de correlação entre as variáveis demográficas e psicográficas com a consciência ecológica. 
A escala desenvolvida e adaptada por Straughan e Roberts (1999) passa, então, a ter trinta itens. Uma questão importante sobre essa escala - e que merece maior aprofundamento - refere-se à sua unidimensionalidade. Apesar de ter sido validada com um só fator, alguns estudos posteriores encontraram na ECCB mais de um fator, como demonstram Ishaswini e Datta (2011), em que a análise fatorial revelou dois fatores, bem como Márcia Grohmann et al. (2012) que encontraram três fatores.

A análise dos itens das escalas sugere que a distinção entre elas está na maior aproximação ou no maior distanciamento entre consciência ambiental e intenção de comportamento de consumo ambiental (ICCA). A escala NEP, que focaliza crenças e valores, é mais abstrata, enquanto a ECCB é, aparentemente, mais indicativa de associação entre consciência ambiental e ICCA. O interessante é que, mesmo assim, é possível encontrar diversos estudos com foco na relação entre consciência ambiental e comportamento de consumo utilizando a NEP em vez da ECCB.

Visto que ainda não se chegou a uma conclusão definitiva sobre o desempenho das duas escalas, este artigo visa refletir qual das duas, NEP ou ECCB, melhor representa a consciência ambiental. Muitos estudos demonstram que uma das consequências da consciência ambiental é o comportamento ambiental. Portanto, sugere-se que a escala que melhor predisser essa associação será mais adequada. A Figura 1 sintetiza essa associação.

Figura 1 - Modelo de comparação adotado na pesquisa
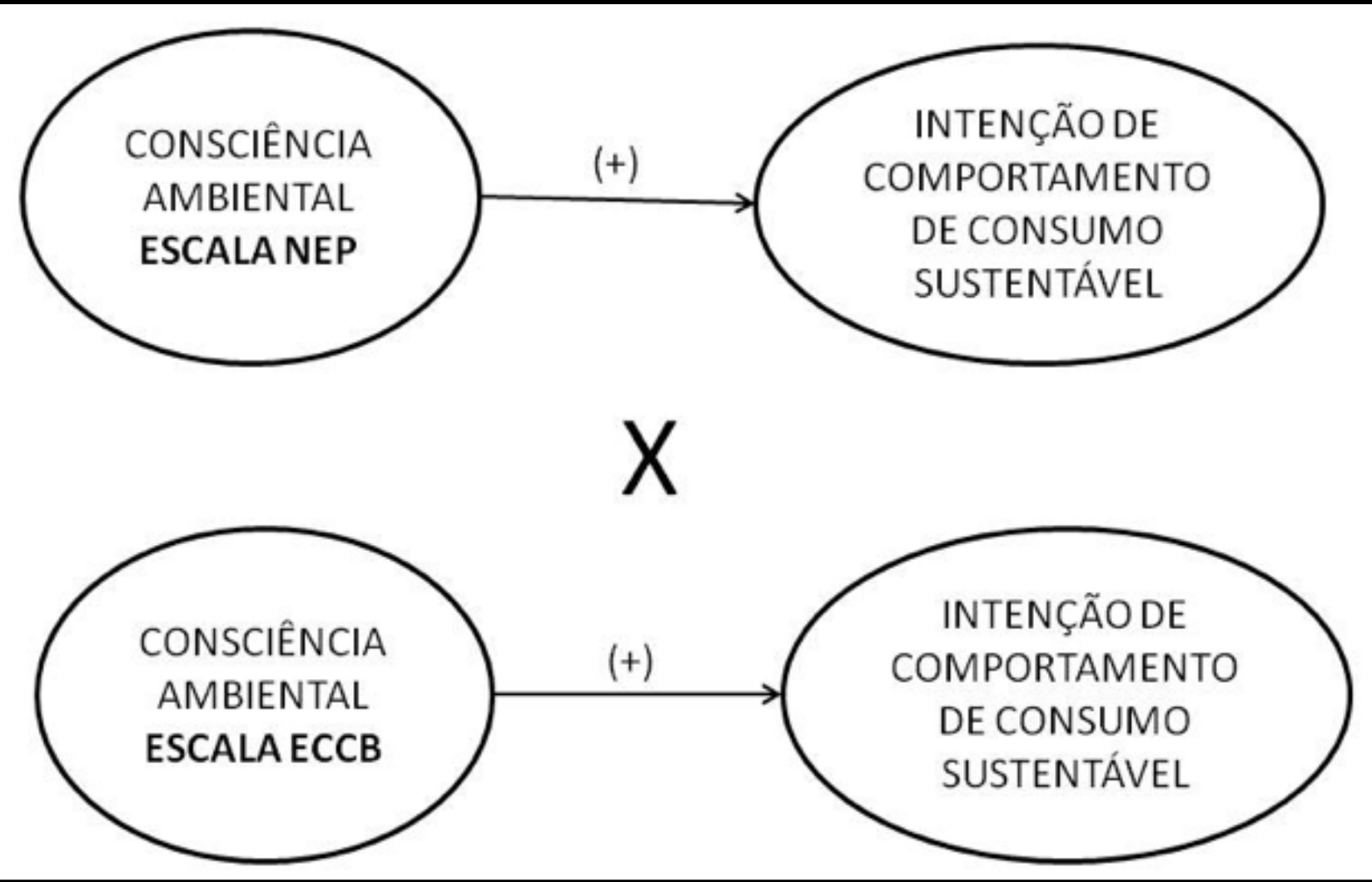

Fonte: Elaborada pelos autores 


\section{METODOLOGIA DE PESQUISA}

Este estudo, quantitativo e de natureza exploratória, abrangeu a avaliação do poder explicativo das escalas NEP e ECCB para medir a consciência ambiental e seu impacto na intenção de comportamento de consumo sustentável (ICCA). Para tanto, foi desenvolvido e operacionalizado um survey com instrumento de coleta de dados enviado e respondido via internet por uma amostra de 538 consumidores em geral de todo território nacional. O disparo e o gerenciamento do link foi administrado durante a primeira semana de março de 2013, por uma empresa especializada em pesquisa de mercado.

Para avaliar a dimensionalidade das escalas, empregou-se a análise fatorial exploratória para as escalas NEP, ECCB e ICCA, a fim de verificar se as variáveis a serem testadas eram função de fatores bem definidos. Para validar as dimensões de cada escala, foram analisadas as validades nomológica, discriminante e de consistência interna, com o emprego da análise de conteúdo dos itens, do coeficiente Alpha de Cronbach e das medidas de correlação entre as escalas somadas de cada dimensão. Para avaliar o efeito das escalas NEP e ECCB na variável dependente ICCA, utilizou-se a técnica de regressão múltipla.

\section{Operacionalização das escalas}

Para a mensuração da consciência ambiental, foram utilizadas as escalas NEP e ECCB. A NEP foi traduzida e validada no contexto brasileiro por Silva Filho et al. (2010). Já a ECCB foi traduzida e validada para o contexto brasileiro por Grohmann et al. (2012), passando a ter 26 itens. Neste artigo, optou-se por utilizar as escalas já traduzidas e validadas.

Como variável dependente, foi utilizada a intenção de comportamento de consumo ambiental (ICCA). Essa escala, composta por nove itens, foi elaborada com base na Environmental behaviors scale, de William Kilbourne e Gregory Pickett (2008), e na escala de comportamento ambiental do estudo de Braga Junior et al. (2012).

\section{Questionário}

Utilizou-se um formulário de pesquisa estruturado contendo quatro seções. A primeira tinha o objetivo de identificar os dados sociodemográficos dos respondentes. A segunda continha as quinze afirmativas da NEP e as 26 da ECCB. Para evitar vieses de resposta, o instrumento de pesquisa foi desenvolvido de maneira que metade dos respondentes visse primeiro a escala NEP e em seguida a ECCB e vice-versa. A terceira seção continha os nove itens para a mensuração da escala ICCA. O Quadro 2 detalha as escalas utilizadas neste estudo. 


\section{Quadro 2 - Escalas utilizadas na pesquisa}

\begin{tabular}{|c|c|c|c|}
\hline \multicolumn{2}{|r|}{ ESCALA NEP } & \multicolumn{2}{|r|}{ ESCALA ECCB } \\
\hline NEP1 & $\begin{array}{l}\text { Nós estamos chegando ao núme- } \\
\text { ro de pessoas que a Terra pode } \\
\text { suportar }\end{array}$ & ECCB1 & $\begin{array}{l}\text { Eu prefiro produtos e alimentos } \\
\text { sem agrotóxicos porque eles } \\
\text { respeitam o meio ambiente. }\end{array}$ \\
\hline NEP2 & $\begin{array}{l}\text { Os seres humanos têm o direito } \\
\text { de modificar o ambiente natural } \\
\text { para atingir suas necessidades }\end{array}$ & ECCB2 & $\begin{array}{l}\text { Eu compro produtos orgânicos } \\
\text { porque são mais saudáveis. }\end{array}$ \\
\hline NEP3 & $\begin{array}{l}\text { Quando os seres humanos in- } \\
\text { terferem na natureza, se produz } \\
\text { freqüentemente conseqüências } \\
\text { desastrosas }\end{array}$ & ECCB3 & $\begin{array}{l}\text { Eu estou disposto(a) a pagar } \\
\text { um pouco mais por produtos e } \\
\text { alimentos que estão livres de } \\
\text { elementos químicos e que pre- } \\
\text { judicam o meio ambiente. }\end{array}$ \\
\hline NEP4 & $\begin{array}{l}\text { A perspicácia humana irá assegu- } \\
\text { rar que nós NÃO faremos a Terra } \\
\text { inabitável }\end{array}$ & ECCB4 & $\begin{array}{l}\text { Quando eu compro produtos } \\
\text { e alimentos as preocupações } \\
\text { com o meio ambiente interfe- } \\
\text { rem na minha decisão de com- } \\
\text { pra. }\end{array}$ \\
\hline NEP5 & $\begin{array}{l}\text { Os seres humanos estão abusan- } \\
\text { do seriamente do meio ambiente }\end{array}$ & ECCB5 & $\begin{array}{l}\text { Eu não compro produtos e } \\
\text { alimentos que podem causar a } \\
\text { extinção de algumas espécies } \\
\text { animais e vegetais. }\end{array}$ \\
\hline NEP6 & $\begin{array}{l}\text { A terra tem riquezas em fontes } \\
\text { naturais, nós temos apenas que } \\
\text { aprendermos a desenvolvê-las }\end{array}$ & ECCB6 & $\begin{array}{l}\text { Eu evito comprar produtos com } \\
\text { embalagens que não são bio- } \\
\text { degradáveis. }\end{array}$ \\
\hline NEP7 & $\begin{array}{l}\text { Plantas e animais têm tanto di- } \\
\text { reito de existir quanto os seres } \\
\text { humanos }\end{array}$ & ECCB7 & $\begin{array}{l}\text { Sempre que possível, eu procu- } \\
\text { ro comprar produtos com em- } \\
\text { balagens reutilizáveis. }\end{array}$ \\
\hline NEP8 & $\begin{array}{l}\text { O equilíbrio natural é suficiente- } \\
\text { mente estável para absorver os } \\
\text { impactos das nações industriais } \\
\text { modernas }\end{array}$ & ECCB8 & $\begin{array}{l}\text { Eu sempre faço um esforço } \\
\text { para reduzir o uso de produ- } \\
\text { tos feitos de recursos naturais } \\
\text { escassos. }\end{array}$ \\
\hline NEP9 & $\begin{array}{l}\text { Apesar de nossas habilidades } \\
\text { especiais, os seres humanos se- } \\
\text { guem sujeitos as leis da natureza }\end{array}$ & ECCB9 & $\begin{array}{l}\text { Eu procuro comprar eletrodo- } \\
\text { mésticos que consomem me- } \\
\text { nos energia. }\end{array}$ \\
\hline NEP10 & $\begin{array}{l}\text { A chamada "Crise Ecológica" que } \\
\text { enfrenta a humanidade tem sido } \\
\text { grandemente exagerada }\end{array}$ & ECCB10 & $\begin{array}{l}\text { Eu sempre tento usar eletrodo- } \\
\text { mésticos (por exemplo: máqui- } \\
\text { na de lavar, chuveiros elétricos } \\
\text { e secadoras) fora do horário de } \\
\text { pico de consumo, que ocorre } \\
\text { entre as } 18 \mathrm{~h} 30 \text { e às } 21 \mathrm{~h} 30 \text {. }\end{array}$ \\
\hline
\end{tabular}




\begin{tabular}{|c|c|c|c|}
\hline NEP11 & $\begin{array}{l}\text { A terra é uma espaçonave com } \\
\text { espaço e fontes muito limitados }\end{array}$ & ECCB11 & $\begin{array}{l}\text { Eu procuro não comprar produ- } \\
\text { tos que possuem grande quan- } \\
\text { tidade de embalagem. }\end{array}$ \\
\hline NEP12 & $\begin{array}{l}\text { O ser humano foi feito para reinar } \\
\text { sobre o resto da natureza }\end{array}$ & ECCB12 & $\begin{array}{l}\text { Quando possível, eu sempre } \\
\text { escolho produtos que causam } \\
\text { menor poluição. }\end{array}$ \\
\hline NEP13 & $\begin{array}{l}\text { O equilíbrio natural é muito deli- } \\
\text { cado e facilmente abalado }\end{array}$ & ECCB13 & $\begin{array}{l}\text { Eu procuro, insistentemente, } \\
\text { reduzir o consumo de energia } \\
\text { elétrica. }\end{array}$ \\
\hline NEP14 & $\begin{array}{l}\text { Os seres humanos irão aprender } \\
\text { o suficiente sobre como a nature- } \\
\text { za funciona para serem capazes } \\
\text { de controlá-la }\end{array}$ & ECCB14 & $\begin{array}{l}\text { Quando eu conheço os pos- } \\
\text { síveis danos que um produto } \\
\text { pode causar ao meio ambiente, } \\
\text { eu não compro este produto. }\end{array}$ \\
\hline NEP15 & $\begin{array}{l}\text { Se as coisas continuarem no cur- } \\
\text { so atual, nós iremos breve expe- } \\
\text { rimentar uma catástrofe ecológica } \\
\text { maior }\end{array}$ & ECCB15 & $\begin{array}{l}\text { Eu já troquei ou deixei de usar } \\
\text { produtos por razões ecológicas. }\end{array}$ \\
\hline \multirow{2}{*}{\multicolumn{2}{|c|}{ COMPORTAMENTO AMBIENTAL }} & ECCB16 & $\begin{array}{l}\text { Na minha residência eu separo } \\
\text { o lixo seco do lixo orgânico. }\end{array}$ \\
\hline & & ECCB17 & $\begin{array}{l}\text { Eu procuro comprar produtos } \\
\text { feitos papel reciclado. }\end{array}$ \\
\hline CA1 & $\begin{array}{l}\text { Sempre que possível, eu compro } \\
\text { produtos ecologicamente corre- } \\
\text { tos. }\end{array}$ & ECCB18 & $\begin{array}{l}\text { Eu já convenci amigos ou pa- } \\
\text { rentes a não comprar produtos } \\
\text { que prejudicam o meio-ambien- } \\
\text { te. }\end{array}$ \\
\hline CA2 & $\begin{array}{l}\text { Sempre que possível, eu compro } \\
\text { alimentos orgânicos }\end{array}$ & ECCB19 & $\begin{array}{l}\text { Eu troquei as lâmpadas da mi- } \\
\text { nha casa por lâmpadas de me- } \\
\text { nor potência para reduzir o con- } \\
\text { sumo de energia elétrica. }\end{array}$ \\
\hline CA3 & $\begin{array}{l}\text { Sempre que possível, utilizo pro- } \\
\text { dutos feitos de material reciclado }\end{array}$ & ECCB20 & $\begin{array}{l}\text { Sempre que possível, eu com- } \\
\text { pro produtos feitos com material } \\
\text { reciclado. }\end{array}$ \\
\hline CA4 & $\begin{array}{l}\text { Eu tento comprar produtos com } \\
\text { embalagens recicláveis }\end{array}$ & ECCB21 & $\begin{array}{l}\text { Quando eu tenho que escolher } \\
\text { entre dois produtos iguais, eu } \\
\text { sempre escolho o que é menos } \\
\text { prejudicial às outras pessoas e } \\
\text { ao meio-ambiente. }\end{array}$ \\
\hline CA5 & $\begin{array}{l}\text { Pagaria mais para comprar pro- } \\
\text { dutos que promovam a proteção } \\
\text { ambiental. }\end{array}$ & ECCB22 & $\begin{array}{l}\text { Eu não compro produtos fabri- } \\
\text { cados ou vendidos por empre- } \\
\text { sas que prejudicam ou desres- } \\
\text { peitam o meio-ambiente. }\end{array}$ \\
\hline
\end{tabular}




\begin{tabular}{|c|c|c|c|}
\hline CA6 & $\begin{array}{l}\text { Pagaria mais para comprar produ- } \\
\text { tos orgânicos. }\end{array}$ & ECCB23 & $\begin{array}{l}\text { Eu comprei lâmpadas mais ca- } \\
\text { ras, mas que economizam mais } \\
\text { energia elétrica. }\end{array}$ \\
\hline CA7 & $\begin{array}{l}\text { Eu sou membro de uma organiza- } \\
\text { ção ambiental. }\end{array}$ & ECCB24 & $\begin{array}{l}\text { Eu tento comprar apenas produ- } \\
\text { tos que podem ser reciclados. }\end{array}$ \\
\hline CA8 & $\begin{array}{l}\text { Eu contribuo financeiramente com } \\
\text { uma organização ambiental. }\end{array}$ & ECCB25 & $\begin{array}{l}\text { Eu normalmente compro o pro- } \\
\text { duto com preço mais baixo, } \\
\text { mesmo sabendo que ele preju- } \\
\text { dica o meio-ambiente. }\end{array}$ \\
\hline CA9 & $\begin{array}{l}\text { Possuo assinatura de uma revista } \\
\text { ambiental. }\end{array}$ & ECCB26 & $\begin{array}{l}\text { Eu não compro produtos para } \\
\text { minha casa que prejudicam o } \\
\text { meio-ambiente. }\end{array}$ \\
\hline
\end{tabular}

Fonte: Elaborado pelos autores.

\section{Amostra}

A amostra se caracterizou como não-probabilística, por conveniência. Foram coletados 644 questionários, dos quais 538 foram considerados válidos. Dos 538 respondentes, 389 (72,8\%) eram do sexo feminino, $248(46,4 \%)$ tinham idade variando entre 25 e 35 anos, $322(60,3 \%)$ possuíam o ensino superior completo e 446 (83\%) declararam salário acima de $R \$ 1.000,00$. Os questionários foram desconsiderados quando se detectou viés de resposta inconsistente e missing values não passíveis de tratamento.

\section{Técnicas de análise}

Os dados foram analisados com duas técnicas estatísticas, a análise fatorial exploratória (AFE) e a regressão linear. A AFE, segundo Joseph F. Hair et al. (2009), analisa as relações entre as variáveis para identificar grupos de itens que formam dimensões latentes. Essa técnica multivariada analisa as correlações entre um grupo de variáveis, com o objetivo de tentar compreender o relacionamento entre elas, ao mesmo tempo em que possibilita, a partir da criação dos fatores, reduzir a quantidade de dados e, assim, facilitar seu estudo e compreensão. Essa técnica foi utilizada para identificar as dimensões, ou grupos de variáveis, que compõem cada figura. $O$ método de rotação dos fatores foi o varimax (Hair et al., 2009)

Além disso, calculou-se o índice KMO, que varia entre zero e um, para analisar o grau de adequação da AFE. Foram adotados os critérios descritos por Hair et al. (2009) para a seleção dos itens integrantes de cada fator, a partir de 0,5. Também foram descartados itens que não tiveram carga fatorial alta ou bem definida, para não comprometer a unidimensionalidade dos fatores. Examinou-se a 
consistência interna de cada fator, pelo emprego do Alpha de Cronbach (doravante representado por a). Depois de selecionar os fatores e verificar sua consistência, foram elaboradas escalas somadas para cada fator. Essa técnica consiste em calcular a média aritmética dos itens presentes em uma escala para então utilizá-la como variável nas análises subsequentes. Para avaliar a validade discriminante, calculou-se a correlação entre as escalas somadas.

Para avaliar a associação entre consciência ambiental e intenção de comportamento de consumo ambiental (ICCA), empregou-se a regressão linear múltipla, sendo considerado o modelo mais ajustado aquele que apresentou o maior poder preditivo, pela avaliação do $R^{2}$ da variável dependente em cada modelo.

\section{DISCUSSÃO DOS RESULTADOS}

Nesta seção serão apresentados os resultados obtidos na presente pesquisa.

\section{Análise fatorial exploratória (AFE)}

Ao analisar a escala NEP, com base nos critérios de extração dos fatores acima descritos, foram extraídos três fatores, os quais correspondem a $61 \%$ da variância, com KMO limítrofe de 0,70. Contudo, durante a análise em função da baixa comunalidade $(<0,5)$, alguns itens da escala foram descartados. Dessa forma, como se verifica na Tabela 1, as três dimensões da escala NEP foram assim denominadas: preocupação com o equilíbrio da natureza; anti-NEP; e limites da natureza. $O$ primeiro fator está relacionado às consequências que a humanidade pode causar ao planeta. O segundo foi composto pelos itens anti-NEP, representando pessoas que não se preocupam com o meio ambiente. As duas variáveis do terceiro fator abordam os limites do meio ambiente. Nenhuma variável apresentou cargas altas para mais de um fator e nenhum dos três fatores apresentou consistência interna boa. A dimensão 1 (equilíbrio), composta por três itens, tem consistência interna satisfatória $(\mathbf{a}=0,72)$. A dimensão anti-NEP apresenta a minimamente requerido de 0,60 ; e a dimensão limites apresenta baixa consistência interna $(\mathbf{a}=0,41)$. Entretanto, segundo Hair et al. (2009), pode-se utilizar uma escala com dois itens quando a correlação de Pearson entre os dois itens for significante ao nível $p<0,01$.

Tabela 1 - Fatores da NEP

\begin{tabular}{|c|l|c|c|c|}
\hline Itens & Afirmativas & $\begin{array}{c}\text { Equilíbrio } \\
\mathbf{\alpha}=\mathbf{0 , 7 2}\end{array}$ & $\begin{array}{c}\text { Anti-NEP } \\
\mathbf{\alpha}=\mathbf{0 , 5 9}\end{array}$ & $\begin{array}{c}\text { Limites } \\
\mathbf{p = 0 , 0 0 0}\end{array}$ \\
\hline NEP3 & $\begin{array}{l}\text { Quando os seres humanos interferem na na- } \\
\text { tureza, se produz freqüentemente conseqüên- } \\
\text { cias desastrosas }\end{array}$ & 0,823 & & \\
\hline
\end{tabular}




\begin{tabular}{|c|c|c|c|c|}
\hline NEP5 & $\begin{array}{l}\text { Os seres humanos estão abusando seriamen- } \\
\text { te do meio ambiente }\end{array}$ & 0,779 & & \\
\hline NEP15 & $\begin{array}{l}\text { Se as coisas continuarem no curso atual, nós } \\
\text { iremos breve experimentar uma catástrofe } \\
\text { ecológica maior }\end{array}$ & 0,766 & & \\
\hline NEP12 & $\begin{array}{l}\text { O ser humano foi feito para reinar sobre o res- } \\
\text { to da natureza }\end{array}$ & & $-0,79$ & \\
\hline NEP8 & $\begin{array}{l}\text { O equilíbrio natural é suficientemente estável } \\
\text { para absorver os impactos das nações indus- } \\
\text { triais modernas }\end{array}$ & & $-0,741$ & \\
\hline NEP2 & $\begin{array}{l}\text { Os seres humanos têm o direito de modificar o } \\
\text { ambiente natural para atingir suas necessida- } \\
\text { des }\end{array}$ & & $-0,681$ & \\
\hline NEP1 & $\begin{array}{l}\text { Nós estamos chegando ao número de pesso- } \\
\text { as que a Terra pode suportar }\end{array}$ & & & $-0,779$ \\
\hline NEP11 & $\begin{array}{l}\text { A terra é uma espaçonave com espaço e fon- } \\
\text { tes muito limitados }\end{array}$ & & & $-0,773$ \\
\hline
\end{tabular}

Fonte: Elaborada pelos autores.

A análise fatorial para a ECCB apresentou uma solução com três dimensões e KMO de 0,94, considerado bastante adequado. Durante o processo de análise da ECCB, algumas variáveis foram retiradas do modelo por apresentarem comunalidades abaixo do recomendável ou ainda por apresentarem altas cargas para mais de um fator, resultando em dezesseis itens. A AFE indicou a formação de três fatores, os quais podem ser vistos na Tabela 2: respeito ao meio ambiente; reciclagem; e economia de energia, O modelo final explicou 59,8\% da variância e nenhuma variável apresentou altas cargas para mais de um fator. Todos os fatores apresentaram consistência interna satisfatória ou boa.

Tabela 2 - Fatores da ECCB

\begin{tabular}{|l|l|c|c|c|}
\hline \multicolumn{1}{|c|}{ Itens } & \multicolumn{1}{|c|}{ Afirmativas } & $\begin{array}{c}\text { Respeito } \\
\mathbf{\alpha = 0 , 8 7}\end{array}$ & $\begin{array}{c}\text { Reciclagem } \\
\mathbf{\alpha = 0 , 8 5}\end{array}$ & $\begin{array}{c}\text { Energia } \\
\mathbf{\alpha = 0 , 6 7}\end{array}$ \\
\hline ECCB26 & $\begin{array}{l}\text { Eu não compro produtos para minha } \\
\text { casa que prejudicam o } \\
\text { meio-ambiente. }\end{array}$ & 0,735 & & \\
\hline ECCB11 & $\begin{array}{l}\text { Eu procuro não comprar produtos } \\
\text { que possuem grande quantidade de } \\
\text { embalagem. }\end{array}$ & 0,696 & & \\
\hline ECCB15 & $\begin{array}{l}\text { Eu já troquei ou deixei de usar pro- } \\
\text { dutos por razões ecológicas. }\end{array}$ & 0,694 & & \\
\hline
\end{tabular}




\begin{tabular}{|c|c|c|c|c|}
\hline ECCB4 & $\begin{array}{l}\text { Quando eu compro produtos e ali- } \\
\text { mentos as preocupações com o } \\
\text { meio ambiente interferem na minha } \\
\text { decisão de compra. }\end{array}$ & 0,656 & & \\
\hline ECCB22 & $\begin{array}{l}\text { Eu não compro produtos fabricados } \\
\text { ou vendidos por empresas que pre- } \\
\text { judicam ou desrespeitam o meio-am- } \\
\text { biente. }\end{array}$ & 0,638 & & \\
\hline ECCB18 & $\begin{array}{l}\text { Eu já convenci amigos ou parentes } \\
\text { a não comprar produtos que prejudi- } \\
\text { cam o meio-ambiente. }\end{array}$ & 0,594 & & \\
\hline ECCB6 & $\begin{array}{l}\text { Eu evito comprar produtos com em- } \\
\text { balagens que não são biodegradá- } \\
\text { veis. }\end{array}$ & 0,538 & & \\
\hline ECCB1 & $\begin{array}{l}\text { Eu prefiro produtos e alimentos sem } \\
\text { agrotóxicos porque eles respeitam o } \\
\text { meio ambiente. }\end{array}$ & 0,536 & & \\
\hline ECCB7 & $\begin{array}{l}\text { Sempre que possível, eu procuro } \\
\text { comprar produtos com embalagens } \\
\text { reutilizáveis. }\end{array}$ & & $-0,808$ & \\
\hline ECCB20 & $\begin{array}{l}\text { Sempre que possível, eu compro } \\
\text { produtos feitos com material recicla- } \\
\text { do. }\end{array}$ & & $-0,769$ & \\
\hline ECCB17 & $\begin{array}{l}\text { Eu procuro comprar produtos feitos } \\
\text { papel reciclado. }\end{array}$ & & $-0,632$ & \\
\hline ECCB24 & $\begin{array}{l}\text { Eu tento comprar apenas produtos } \\
\text { que podem ser reciclados. }\end{array}$ & & $-0,625$ & \\
\hline ECCB21 & $\begin{array}{l}\text { Quando eu tenho que escolher entre } \\
\text { dois produtos iguais, eu sempre es- } \\
\text { colho o que é menos prejudicial às } \\
\text { outras pessoas e ao meio-ambiente. }\end{array}$ & & $-0,613$ & \\
\hline ECCB23 & $\begin{array}{l}\text { Eu comprei lâmpadas mais caras, } \\
\text { mas que economizam mais energia } \\
\text { elétrica. }\end{array}$ & & & 0,793 \\
\hline ECCB19 & $\begin{array}{l}\text { Eu troquei as lâmpadas da minha } \\
\text { casa por lâmpadas de menor potên- } \\
\text { cia para reduzir o consumo de ener- } \\
\text { gia elétrica. }\end{array}$ & & & 0,722 \\
\hline ECCB9 & $\begin{array}{l}\text { Eu procuro comprar eletrodomésti- } \\
\text { cos que consomem menos energia. }\end{array}$ & & & 0,721 \\
\hline
\end{tabular}

Fonte: Elaborado pelos autores. 
A AFE realizada para a intenção de comportamento de consumo ambiental (ICCA) indicou a formação de dois fatores, correspondentes a $64,8 \%$ da variância total explicada, com KMO adequado $(0,80)$. Nenhuma variável apresentou altas cargas para mais de um fator e, diferentemente das análises anteriores, todos os itens da escala permaneceram no modelo. Conforme se mostra na Tabela 3, os dois fatores resultantes da análise foram denominados consumo sustentável - englobando itens referentes especificamente ao consumo - e engajamento - relacionado ao comportamento de participação e envolvimento em questões ambientais.

Tabela 3 - Fatores do comportamento ambiental

\begin{tabular}{|c|c|c|c|}
\hline Itens & Afirmativas & $\begin{array}{c}\text { Comsumo } \\
\alpha=0,86\end{array}$ & $\begin{array}{c}\text { Engajamento } \\
\alpha=0,79\end{array}$ \\
\hline CA4 & $\begin{array}{l}\text { Eu tento comprar produtos com emba- } \\
\text { lagens recicláveis }\end{array}$ & 0,819 & \\
\hline CA1 & $\begin{array}{l}\text { Sempre que possível, eu compro pro- } \\
\text { dutos ecologicamente corretos. }\end{array}$ & 0,812 & \\
\hline CA3 & $\begin{array}{l}\text { Sempre que possível, utilizo produtos } \\
\text { feitos de material reciclado }\end{array}$ & 0,806 & \\
\hline $\mathrm{CA} 2$ & $\begin{array}{l}\text { Sempre que possível, eu compro ali- } \\
\text { mentos orgânicos }\end{array}$ & 0,762 & \\
\hline CA6 & $\begin{array}{l}\text { Pagaria mais para comprar produtos } \\
\text { orgânicos. }\end{array}$ & 0,73 & \\
\hline CA5 & $\begin{array}{l}\text { Pagaria mais para comprar produtos } \\
\text { que promovam a proteção ambiental. }\end{array}$ & 0,727 & \\
\hline CA7 & $\begin{array}{l}\text { Eu sou membro de uma organização } \\
\text { ambiental. }\end{array}$ & & 0,855 \\
\hline CA8 & $\begin{array}{l}\text { Eu contribuo financeiramente com uma } \\
\text { organização ambiental. }\end{array}$ & & 0,847 \\
\hline CA9 & $\begin{array}{l}\text { Possuo assinatura de uma revista am- } \\
\text { biental. }\end{array}$ & & 0,822 \\
\hline
\end{tabular}

Fonte: Elaborada pelos autores

\section{Comparação das escalas}

Utilizando as escalas somadas de cada fator, valemo-nos da regressão linear múltipla para avaliar qual escala - NEP ou ECCB - melhor explica a intenção de comportamento de consumo ambiental (ICCA), sendo que a regressão foi feita a partir de cada fator. Atestou-se a validade discriminante dos fatores pela avaliação da correlação de Pearson entre as escalas somadas que refletem as dimensões da NEP, ECCB e intenção de comportamento de consumo ambiental (ICCA). Não foram detectadas correlações altas entre as dimensões que indicam a necessidade de reformulação dos fatores, havendo confirmação da validade discriminante. 
Conforme exposto na Tabela 4, as quatro regressões apresentaram coeficientes significantes. Não foram encontrados indícios de multicolinearidade entre as variáveis independentes. Os valores de VIF apresentaram-se adequados e abaixo de cinco (Hair et al., 2009). Analisando as dimensões das escalas, verifica-se que, para a relação proposta entre NEP e ICCA, há uma associação positiva entre as dimensões "preocupação com o equilíbrio" e "limites da natureza" e o "comportamento de consumo ambiental", e negativa, conforme esperado, para a associação entre a variável resposta e a dimensão anti-NEP.

Os resultados foram inconsistentes para a relação entre as dimensões da NEP e o "engajamento". Os "limites da natureza" apresentaram associação positiva com o "engajamento". Surpreendentemente, o fator "equilíbrio da natureza" apresentou uma relação negativa e o anti-NEP, uma relação positiva.

Tabela 4 - Análise da relação entre consciência ambiental e comportamento

\begin{tabular}{|c|c|c|c|c|c|c|c|c|}
\hline $\begin{array}{l}\text { Escalas de } \\
\text { consciência }\end{array}$ & \multicolumn{4}{|c|}{$\begin{array}{l}\text { Comportamento de } \\
\text { consumo ambiental }\end{array}$} & \multicolumn{4}{|c|}{ Engajamento } \\
\hline NEP & Coef & DesvPad & $\mathbf{P}$ & $\mathbf{R}^{2}$ & Coef & DesvPad & $\mathbf{P}$ & $\mathbf{R}^{2}$ \\
\hline $\begin{array}{l}\text { Equilíbrio da } \\
\text { ntureza }\end{array}$ & 0,21 & 0,04 & 0,00 & $7,0 \%$ & $-0,16$ & 0,05 & 0,00 & $6,6 \%$ \\
\hline Anti-NEP & $-0,1$ & 0,04 & 0,01 & & 0,21 & 0,04 & 0,00 & \\
\hline $\begin{array}{l}\text { Limites da } \\
\text { natureza }\end{array}$ & 0,08 & 0,03 & 0,02 & & 0,07 & 0,04 & 0,052 & \\
\hline Constante & 2,59 & 0,24 & 0,00 & & 1,93 & 0,27 & 0,00 & \\
\hline ECCB & Coef & DesvPad & $P$ & $R^{2}$ & Coef & DesvPad & $\mathrm{P}$ & $\mathrm{R}^{2}$ \\
\hline $\begin{array}{l}\text { Respeito ao } \\
\text { meio ambiente }\end{array}$ & 0,45 & 0,04 & 0,00 & $60,2 \%$ & 0,32 & 0,05 & 0,00 & $6,9 \%$ \\
\hline Reciclagem & 0,39 & 0,04 & 0,00 & & N.S. & 0,05 & 0,00 & \\
\hline $\begin{array}{l}\text { Economia de } \\
\text { eergia }\end{array}$ & N.S. & & & & $-0,26$ & & & \\
\hline Constante & 0,56 & 0,1 & 0,00 & & 2 & 0,22 & 0,00 & \\
\hline
\end{tabular}

Fonte: Elaborada pelos autores.

As dimensões da ECCB "respeito ao meio ambiente" e "reciclagem" apresentaram uma relação significante e significativa $\left(R^{2}\right.$ de $\left.60,2 \%\right)$ com o comportamento de consumo ambiental, indicando que pessoas preocupadas com o meio ambiente e com interesse sobre reciclagem são mais propensas ao consumo sustentável. Para o "engajamento", o fator "respeito ao meio ambiente" foi significante e positivamente relacionado, mas a "reciclagem" não foi significante e a "economia de energia" apresentou uma relação inversa ao engajamento. Sugere-se que os respondentes, quando pensam em economia de energia, visam a outros benefícios, provavelmente econômicos, e não associam necessariamente economia de energia à preservação do meio ambiente. 
Ao examinar os valores de $R^{2}$ das regressões, percebe-se que a escala NEP foi capaz de explicar somente $6,6 \%$ da variância do "engajamento" e 7,0\% do "comportamento de consumo ambiental", o que é pouco significativo. Já a escala ECCB explica somente $6,9 \%$ do "engajamento", pouco significativo, porém explica $60,2 \%$ do "comportamento de consumo ambiental". Uma primeira constatação é que ambas não conseguiram dar um bom poder explanatório à dimensão engajamento da ICCA. Ao se verificar os itens componentes deste fator, pode se inferir que esse resultado seja decorrente do fato de as manifestações de comportamento ambiental no Brasil não se darem por meio das ações indicadas nos itens constantes do "engajamento".

Esses resultados também podem estar relacionados às próprias características das escalas. A NEP apresenta itens de natureza mais abstrata, formado por crenças, muitas vezes distantes das práticas de consumo. Depreende-se que seu baixo poder explanatório é decorrente da existência de fatores mediadores, como interesses e atitudes, que são consequentes das crenças (Bagozzi, 1992) e que deveriam ser considerados como antecedentes da intenção de comportamento de consumo. Portanto, uma pessoa que apresente um alto nível de consciência ambiental mensurada por meio da NEP não necessariamente terá a intenção de comportamento de consumo ambiental.

De toda maneira, a ECCB demonstrou ter maior poder explicativo e, portanto, indica-se ser mais adequada que a NEP para se mensurar a consciência ambiental nos estudos de comportamento do consumidor.

\section{CONSIDERAÇÕES FINAIS}

Entre os pesquisadores, ainda não há um consenso sobre qual é o melhor método de se mensurar a consciência ambiental, sendo possível encontrar diferentes escalas em uso. Portanto, este trabalho teve como objetivo comparar duas escalas de mensuração do nível de consciência ambiental. Empregando a escala NEP, uma das mais utilizadas pelos autores, inclusive nos estudos de comportamento de consumo, e a escala ECCB, formulada especificamente para o consumo, buscou-se avaliar empiricamente qual representaria melhor a consciência ambiental em relação ao comportamento dos consumidores.

Com base nos resultados, as duas escalas foram validadas e mostraram ser compostas por três fatores cada. A ECCB se mostrou mais adequada para mensurar a consciência ambiental nos estudos de comportamento de consumo, apresentando um $R^{2}$ de $60,2 \%$. A NEP parece ser uma das escalas preferidas, inclusive nos estudos de comportamento de consumo. Contudo, os resultados encontrados no presente artigo indicam o seu baixo poder explicativo, principalmente quando comparados com os da ECCB.

A NEP apresenta itens de natureza mais abstrata, formado por crenças, muitas vezes distantes das práticas de consumo. Depreende-se que seu baixo poder explanatório é decorrente da existência de 
fatores mediadores, como interesses e atitudes, que são consequentes das crenças e que deveriam ser considerados como antecedentes da intenção de comportamento de consumo. Desde o processo de revisão teórica, passando pelo tratamento estatístico dos dados e pela análise dos resultados, constatou-se a maior propriedade da escala ECCB para explicar intenção de comportamento de consumo ambiental, por conter em suas medidas itens mais próximos do cotidiano das pessoas.

Como decorrência desta análise, recomenda-se que, para estudos futuros de comportamento de consumo ambiental no Brasil, a escala ECCB seja a mais utilizada, por ser a mais adequada.

Entre as limitações da pesquisa encontram-se o processo de coleta de dados, feito de maneira não-probabilística por conveniência, o que impede a generalização dos resultados obtidos. Em função da revisão da literatura, decidiu-se trabalhar com a intenção de comportamento de consumo ambiental como variável dependente. Entretanto, sugere-se que estudos futuros considerem a inclusão de outras variáveis como atitude e associações, para aumentar o poder preditivo do modelo.

Depreende-se do estudo que os profissionais de comunicação corporativa de empresas que consideram a preocupação com o meio ambiente como valor de marca, ao avaliar o brand equity, façam uso da ECCB, por seu melhor desempenho em predizer a intenção de comportamento de consumo ambiental.

\section{REFERÊNCIAS}

AKEHURST, Gary; AFONSO, Carolina; GONÇALVES, Helena Martins. Re-examining green purchase behavior and the green consumer profile: new evidences. Management Decision, v. 50, n. 5, p. 972-988, 2012.

ARNOCKY, Steven; DUPUIS, Darcy; STROINK, Mirella L. Environmental concern and fertility intentions among Canadian university students. Population Environment, v. 34, p. 279-292, 2012.

BAGOZZI, Richard P. The self-regulation of attitudes, intentions, and behavior. Social Psychology Quarterly, v. 55, n. 2, p. 178-204, jun. 1992.

BEDANTE, Gabriel N. A influência da consciência ambiental e das atitudes em relação ao consumo sustentável na intenção de compra de produtos ecologicamente embalados - Porto Alegre. Dissertação (Mestrado em Administração) - Universidade Federal do Rio Grande do Sul 2004.

BLAKE, Donald. E.; GUPPY, Neil; URMETZER, Peter. Canadian public opinion and environmental action: evidence from British Columbia. Canadian Journal of Political Science, v. 30, p. 451-472, 1997.

BRAGA JUNIOR, Sérgio S. et al. Uma análise da consciência ecológica para o consumo "verde" no varejo supermercadista. Revista de Gestão Social e Ambiental, v. 6, n. 2, p. 134-148, 2012.

CASEY, Paul J.; SCOTT, Kylie. Environmental concern and behaviour in an Australian sample within an ecocentric-anthropocentric framework. Australian Journal of Psychology, v. 58, p. 57-67, 2006. 
CERVELLON, Marie-Cécile. Victoria's dirty secrets: effectiveness of green not-for-profit messages targeting brands. Journal of Advertising, v. 41, n. 4, p. 133-145, 2012.

CHUNG, Shan-Shan; POON, Chi Sun A comparison of waste reduction practices and the new environmental paradigm in four southern Chinese areas. Environmental Management, v. 26, p. 195-206, 2000.

DUNLAP, Riley E. et al. Measuring endorsement of the new ecological paradigm: a revised NEP scale. Journal of Social Issues, v. 56, p. 425-442, 2000.

EBREO, Angela; HERSHEY, James; VINING, Joanne. Reducing solid waste. linking recycling to environmental responsible consumerism. Environment and Behavior, v. 31, p. 107-135, 1999.

FUJII, Satoshi. Environmental concern, attitude toward frugality, and ease of behavior as determinants of pro-environmental behavior intentions. Journal of Environmental Psychology, v. 26, p. 262-268, 2006.

$\mathrm{GOOCH}$, Geoffrey D. Environmental beliefs and attitudes in Sweden and the Baltic states. Environment and Behavior, v. 27, p. 513-539, 1995.

GROHMANN, Márcia Zampieri et al. Comportamento ecologicamente consciente do consumidor: adaptação da escala ECCB para o contexto brasileiro. Revista de Gestão Social e Ambiental, v. 6, n. 1, p. 102-116, 2012.

HAIR, Joseph F. et al. Análise multivariada de dados. 6. ed. Porto Alegre: Bookman, 2009.

HARTMANN, Patrick; APAOLAZA-IBÁÑEZ, Vanessa. Consumer attitude and purchase intention toward green energy brands: the roles of psychological benefits and environmental concern. Journal of Business Research, v. 65, p. 1254-1263, 2012.

HIRSH, Jacob B. Personality and environmental concern. Journal of Environmental Psychology, v. 30, p. 245-248, 2010.

ISHASWINI, None.; DATTA, Saroj Kumar. Pro-environmental concern influencing green buying: a study on Indian consumers. International Journal of Business and Management, v. 6, n. 6, p. 124-133, 2011.

JAIN, Sanjay K.; KAUR, Gurmeet. Role of socio-demographics in segmenting and profiling green consumers. Journal of international Consumer Marketing, v. 18, n. 3, p. 107-146, 2006.

KAISER, Florian G.; HUBNER, Gundula; BOGNER, Franz X. Contrasting the theory of planned behavior with the valuebelief-norm model in explaining conservation behavior. Journal of Applied Social Psychology, v. 35, p. 2150-217, 2005.

KILBOURNE, William; PICKETT, Gregory. How Materialism affects environmental beliefs, concern, and environmentally responsible behavior. Journal of Business Research, v. 61, p. 885-893, 2008.

MAINIERI, Tina et al. Green buying: the influence of environmental concern on consumer behavior. Journal of Social Psychology, v. 137, p. 189-204, 1997.

MANN, Horace et al. Drivers of sustainable supply chain management. The IUP Journal of Operations Management, v. 9, n. 4 , p. 52-63, 2010.

MATOS, Stelvia; HALL, Jeremy. Integrating sustainable development in the supply chain: the case of life cycle assessment in oil and gas and agricultural biotechnology. Journal of Operations Management, v. 25, n. 6, p. 1083-1102, 2007.

MOSTAFA, Mohamed M. Gender differences in Egyptian consumers' green purchase behaviour: the effects of environmental knowledge, concern and attitude. International Journal of Consumer Studies, v. 31, p. 220-229, 2007. 
NATALI, Ubiratan de Castro. A influência da consciência ambiental a das atitudes em relação ao consumo na intenção de educadores de ensino superior de utlização de sacolas plásicas na cidade de Belo Horizonte. 2012. 146 f. Dissertação (Mestrado em Administração) - Faculdades Pedro Leopoldo, Pedro Leopoldo (MG), 2012.

NISBET, Elizabeth K.; ZELENSKI, John M.; MURPHY, Steven A. The nature relatedness scale: linking individuals' connection with nature to environmental concern and behavior. Environment and Behavior, v. 41, n. 5, p. 715-740, 2009.

POORTINGA, Wouter; STEG, Linda; VLEK, Charles. Environmental risk concern and preferences for energy-saving measures. Environment and Behavior, v. 34, n. 4, p. 455-478, 2002.

Values, environmental concern, and environmental behavior: a study into household energy use. Environment and Behavior, v. 36, n. 1, p. 70-93, 2004.

RILEY, Lynn Sudbury; KOHLBACHER, Florian; HOFMEISTER, Agnes. A cross-cultural analysis of pro-environmental consumer behavior among seniors. Journal of Marketing Management, v. 28, n. 3-4, p. 290-312, 2012.

ROBERTS, James A. Green consumers in the 1990: profile and implications for advertising. Journal of Business Research, v. 36, p. 217-231, 1996.

ROBERTS, James A.; BACON, Donald R. Exploring the subtle relationship between environmental concern and ecologically conscious consumer behavior. Journal of Business Research, v. 40 p. 79-89, 1997.

SAID, Aini Mat et al. Environmental concerns, knowledge and practices gap among Malaysian teachers. International Journal of Sustainability in Higher Education, v. 4, n. 4, p. 305-313, 2003.

SANTOS, Thaís Chacon dos et al. Movimento do consumo consciente: do cidadão consumidor ao consumidor cidadão? In: ENCONTRO DE ADMINISTRAÇÃO PÚBLICA E GOVERNANÇA, 2008, Salvador. Anais... Salvador: EnAPG, 2008.

SCHLEGELMILCH, Bodo B.; BOHLEN, Greg M.; DIAMANTOPOULOS, Adamantios. The link between green purchasing decisions and measures of environmental consciousness. European Journal Marketing, v. 30, n. 5, p. 35-55, 1996.

SCHULTZ, P. Wesley.; OSKAMP, Stuart. Effort as a moderator of the attitude-behavior relationship: general environmental concern and recycling. Social Psychology Quarterly, v. 59, p. 375-383, 1996.

SCOTT, David; WILLITS, Fern K. Environmental attitudes and behavior. Environment and Behavior, v. 26, p. 239-260, 1994.

SILVA FILHO, JOSÉ Carlos L. da et al. Estudo sobre o novo paradigma ecológico (NPE) no Brasil: medindo a consciência ambiental através da escala NEP. In: EnANPAD, 34, . 2010, Rio de Janeiro. Anais... Rio de Janeiro: Anpad, 2010.

SINGH, Narendra; GUPTA, Kamika. Environmental attitude and ecological behavior of Indian consumers. Social Responsibility Journal, v. 9, n. 1, p. 4-18, 2013.

SOUZA, Renato Santos de. Evolução e condicionantes da evolução da gestão ambiental nas empresas. REAd - Edição Especial 30, v. 8, n. 6, nov.-dez 2002.

STRAUGHAN, Robert D.; ROBERTS, James A. Environmental segmentation alternatives: a look at green consumer behavior in the new millennium. Journal of Consumer Marketing, v. 16, n. 6, p. 558-575, 1999.

THAPA, Brijesh. Environmentalism: the relation of environmental attitudes and environmentally responsible behaviors among undergraduate students. Bulletin of Science, Technology, \& Society, v. 19, p. 426-438, 1999. 
VINING, Joanne; EBREO, Angela. Predicting recycling behavior from global and specific environmental attitudes and changes in recycling opportunities. Journal of Applied Social Psychology, v. 22, p. 1.580-1.607, 1992.

ZURAIDAH, Ramly et al. Environmental conscious behaviour among male and female Malaysian consumers. International Journal of Sustainable Development, v. 4, n. 8, p. 55-64, 2012.

Recebido em: 01.03.2013 / Aceito em: 21.06.2013 